Cahiers $d u$ MONDE RUSSE

\section{Cahiers du monde russe}

Russie - Empire russe - Union soviétique et États indépendants

45/3-4 | 2004

Varia

\title{
Julie Hessler, A social history of Soviet trade
}

\section{Alessandro Stanziani}

\section{OpenEdition \\ Journals}

Édition électronique

URL : https://journals.openedition.org/monderusse/4198

DOI : 10.4000/monderusse.4198

ISSN : $1777-5388$

\section{Éditeur}

Éditions de l'EHESS

\section{Édition imprimée}

Date de publication : 1 juillet 2004

Pagination : 690-692

ISBN : 2-7132-2009-2

ISSN : $1252-6576$

\section{Référence électronique}

Alessandro Stanziani, « Julie Hessler, A social history of Soviet trade», Cahiers du monde russe [En ligne], 45/3-4 | 2004, mis en ligne le 03 juin 2009, consulté le 03 septembre 2022. URL : http:// journals.openedition.org/monderusse/4198; DOI : https://doi.org/10.4000/monderusse.4198

Ce document a été généré automatiquement le 3 septembre 2022

Tous droits réservés 


\title{
Julie Hessler, A social history of Soviet trade
}

\author{
Alessandro Stanziani
}

\section{RÉFÉRENCE}

JUlie HESSLER, A social history of Soviet trade. Trade policy, retail practices and consumption, 1917-1953. Princeton-Oxford, Princeton University Press, 2004,

XVI-366 p.

1 Disons-le d'emblée : cet ouvrage est extraordinaire à la fois par le sujet abordé et la manière dont il est traité, par le style et les sources mobilisées. Il comble une lacune importante de l'historiographie de l'ex-URSS avec une finesse et une profondeur qui le mettent au niveau des meilleurs ouvrages d'histoire économique et sociale. Julie Hessler, jeune historienne de l'université de l'Oregon, maîtrise parfaitement aussi bien la bibliographie que les archives, très peu exploitées jusqu'à présent, sur le commerce en URSS et, qui plus est, sur une période longue et complexe qui va de la révolution jusqu’à la mort de Stalin.

Chaque chapitre s'organise autour d'un axe chronologique et problématique et comprend une introduction claire et des conclusions partielles qui permettent au lecteur de saisir la force et la nouveauté des arguments avancés. Ainsi, après avoir évoqué l'organisation du commerce et les politiques commerciales dans la Russie tsariste et pendant la Première Guerre mondiale, Julie Hessler détaille « l'invention du socialisme » à l'époque de la guerre civile, notamment en matière de réseaux de distribution, de cartes de rationnement et de calcul des quantités à allouer. Les critères sous-jacents à ces calculs, ainsi que leur mise en pratique, sont explicités. Ce même chapitre fait état des réformes des années 1920, de la mise en place d'un véritable réseau d'agents commerciaux soviétiques et de la transformation des coopératives commerciales. Le troisième chapitre ("Shopkeepers and the state ») étudie les années 1920 du point de vue des marchands et des consommateurs. Il détaille les stratégies des commerçants dans un 
milieu politique hostile ainsi que les différenciations sociales grandissantes, notamment via l'accès aux biens de consommation.

3 La fin de la NEP, les conflits sur ce sujet entre les différents commissariats et les mesures adoptées sont étudiés dans le chapitre suivant. Une fois de plus, la pénurie des denrées alimentaires, jusqu'à la famine, constitue le déclencheur, mi-fondé, mi-inventé, de ce virage. Les formes de rationnement, à la fois anciennes et nouvelles, et la corruption qu'elles entraînent sont analysées en détail, tout comme (et c'est là une innovation historiographique très importante) la redécouverte du marché, de son mécanisme et surtout de la "qualité des produits " pendant les années 1930. Ce nouveau paradigme des politiques commerciales et des modèles de consommation - s'effondre avec la guerre. Ainsi les bazars remplacent les marchés alors que le rationnement et le plan sont renforcés ; l'après-guerre sera caractérisé par l'ambition de "normaliser » le commerce (multiplication des produits, création de grands magasins, retour au marché et aux contrats de vente, etc.), mais tout en conservant les mesures administratives visant à différencier l'accès aux biens suivant le groupe socio-politique d'appartenance.

Pour toute la période couverte, l'auteur montre l'imbrication entre crise économique et réforme économique, entre plan et marché - et cela même à des époques (années 1930, Deuxième Guerre mondiale et après-guerre) où l'on ne se serait pas attendu à ce mélange typique de la NEP. L'auteur dépasse ainsi les clivages méthodologiques traditionnels entre histoire idéologique et histoire économique et, surtout, entre analyse macroéconomique et sociale et cas individuels. Si l'analyse macro ne constitue ni la toile de fond ni le simple résultat des cas individuels, ces derniers gardent leur unicité tout en étant intégrés au sein d'une mouvance générale. De quoi réjouir à la fois les adeptes de la micro-histoire et ceux de la nouvelle histoire sociale.

5 Chose plus rare encore parmi les spécialistes de l'URSS, l'auteur n'hésite pas à questionner la spécificité du système étudié. Elle peut ainsi mettre en évidence les éléments communs à l'URSS et à d'autres pays européens, notamment pendant les guerres mondiales, mais aussi leur décalage, lié en partie à l'idéologie et aux perceptions soviétiques, en partie aux conditions structurelles de cette économie. À partir de ces prémisses, Hessler remet en question la thèse selon laquelle l'époque staliniste serait caractérisée par des différences grandissantes, au niveau des salaires et des conditions de vie, entre cadres et ouvriers. Au contraire, ce seraient les conditions de vie urbaines qui auraient pris le pas sur celles des campagnes. Dans ce contexte, l'attention accordée par les dirigeants soviétiques, Stalin en tête, à l'essor des produits « de luxe », mérite d'être prise au sérieux.

6 Cet ouvrage incontournable, qui constituera désormais une référence obligée pour tout historien de l'économie et de la société soviétique, ne laisse que peu de place à la critique. On aurait plutôt envie de questionner l'auteur sur deux ou trois points qui relèvent à la fois de la méthode et du contenu. Ils portent sur les notions de luxe et de pénurie. Ainsi, il est sans doute intéressant de suivre l'auteur dans sa démonstration quant à la présence, voire même au soutien, apporté aux produits de luxe, à des époques comme les années 1930 ou l'après-guerre. Cependant, on ne sait pas clairement si c'est l'auteur ou ses sources - et lesquelles? - qui classe ces produits comme étant de luxe. Comme les spécialistes de l'histoire des statistiques (Jean-Claude Perrot en tête), de l'histoire de l'Ancien Régime en France et de la consommation en général le savent bien, la définition du luxe ne va pas de soi mais constitue un enjeu intellectuel, politique et social majeur. Le même produit (et surtout ses différentes variétés) peut être jugé 
comme étant de luxe à un moment donné, et ordinaire peu après (ou inversement, comme en période de guerre). Cet argument est d'autant plus important en Russie-URSS que la définition des produits de luxe y est liée à une association entre certains produits et des classes sociales particulières (nobles, "bourgeois", commerçants, contrerévolutionnaires, etc.).

7 On peut avancer le même argument en ce qui concerne la pénurie d'un produit : celui-ci peut être d'accès limité en termes monétaires ou en termes réels alors que, dans un cas comme dans l'autre, sa raréfaction ne frappe pas de la même manière les différentes couches de la population. Dans le livre de Hessler, on oscille entre une pénurie considérée comme " objective » et une pénurie relative, liée à la fois à l'acteur qui parle et à ses perceptions. Ce problème est d'autant plus important qu'en URSS, la limite entre la "pénurie » et la «famine» pose toujours problème: les dirigeants (tsaristes comme soviétiques) nient toujours l'occurrence des famines et, comme le montre Julie Hessler, ils interprètent souvent la pénurie comme le résultat d'une demande excessive plutôt que d'une offre insuffisante...

8 Ces considérations renvoient à un seul et même problème, à savoir la difficulté à mobiliser pour l'URSS des catégories socio-économiques générales qui, si elles rendent d'une part la comparaison possible, risquent d'autre part d'affaiblir toute possibilité de saisir la spécificité de ce système, surtout si elles ne sont pas questionnées. Mais, de ce point de vue aussi, le livre de Hessler fera sans doute figure de précurseur. 